II.

\title{
Die anatomische und klinische Grundlage des orthopädischen Korsetts.
}

\author{
Von \\ Dr. Wilhelm Becker, \\ Spezialarzt für Orthopädie in Bremen.
}

Mit 3 Abbildungen.

Wenn zur Erreichung irgend eines Zieles eine grosse Zahl von Mitteln angegeben wird, so lässt sich aus diesem Umstande mit Sicherheit schliessen, dass auch nicht eines derselben seinen $Z$ weck jemals voll und ganz erreicht hat. Denn sonst wïrde eben jedermann diesem einen Mittel anhangen und alle anderen hintansetzen.

Es gibt wohl in der ganzen Medizin kaum ein anderes therapeutisches Rüstzeug, das in so vielen Variationen und Modifikationen vertreten wäre als das orthopädische Korsett, d. h. diejenige Rumpfbandage, welche auf irgend eine Weise die Wirbelsäule fixieren, entlasten, extendieren oder redressieren soll. Von der Wahl des Materials sehe ich dabei ganz ab, denn es ist absolut belanglos, ob hierzu Gips, Holz, Pappe, Wasserglas, Drell, Leder, Cellulose, Celluloid, Filz, Aluminium etc. gewählt wird, und man kann schlechthin sagen, dass es nur wenige Materialien gibt, die nicht schon einmal zu Korsetts verarbeitet worden wären. Aber wenn wir von dieser rein technischen Frage absehen, so drängt sich uns noch eine andere, viel wichtigere Mannigfaltigkeit auf, wenn wir die Art der Konstruktion betrachten, d. h. die mechanischen Prinzipien, die dem Bau dieser Wirbelsäulengerüste zu grunde liegen. Wollen wir uns über den Wert oder Unwert derselben ein klares Bild machen, so gibt es hierzu nur einen einzigen Weg: die Betrachtung der Wirbelsäule rom anatomisch-mechanischen Standpunkt aus. Als Resultat unserer Überlegungen wird sich dann von selbst die Beantwortung der klinisch-wichtigsten Fragen der Korsettherapie ergeben, nämlich die der Indikationsstellung sowie die der darauf beruhenden Konstruktion.

Wie der Mast aus dem Schiffsrumpfe hervorragt, so wächst gewissermassen die Wirbelsäule aus dem Becken hervor; und wie jener durch die 
Schiffstaue, so wird diese durch Muskelzug im Gleichgewicht erhalten. Bekanntlich ist ihr Sakralteil nicht ankylotisch mit dem Beckenring verwachsen, aber die Verbindung durch zahlreiche starke Bänder ist doch eine so straffe, dass der Effekt derselbe ist. Hieraus ergibt sich ohne weiteres, dass der Beckenring, zumal die Darmbeincrista, einen äusserst günstigen Angriffspunkt für das untere Ende der Wirbelsäule darstellt, und das ist für unsere Betrachtungen der wichtigste Punkt, in dem sich Beckenring und Schulterring voneinander unterscheiden. Der ganze übrige Abschnitt der Wirbelsäule ist frei beweglich und bietet in dreifacher Weise seiner mechanischen Inangriffnahme die denkbar ungünstigsten Verhältnisse dar:

1. er wird stark belastet durch den auf dem oberen Ende frei balancierenden Kopf:

2. er trägt in seinem mittleren Abschnitt den durch Rippenreifen gebildeten Brustkorb;

3. er liegt derartig im Körper, dass er nur von einzigen Seite, nämlich von hinten, den therapeutischen Bemühungen direkt zugänglich ist.

Der erste Punkt ist von Bedeutung, wenn es gilt, die Wirbelsäule vom Gewicht des Kopfes zu entlasten. Wir müssen uns darüber klar werden, dass dies nur möglich wäre, wenn uns der Kopf entsprechende Angriffspunkte darböte. Dies ist aber nur teilweise der Fall, nämlich am Hinterhaupt; der Unterkiefer dagegen würde bei einer wirklichen Entlastung fest gegen den Oberkiefer gepresst und somit jede Nahrungsaufnahme und jedes Sprechen unmöglich werden. In richtiger Würdigung dieser Tatsache greift $\mathrm{Wullstein}{ }^{1}$ ) bei seinen extendierenden Korsetts den Kopf nicht am Kinn und Hinterhaupt, sondern an Stirn und Hinterhaupt, also im fronto-occipitalen Durchmesser, an, wobei ,nach Möglichkeit die oberen Orbitalränder ausmodelliert werden“, um ein Abrutschen nach unten-vorn zu vermeiden. Diese Methode ist jedenfalls anatomisch richtig ausgedacht; ihre Nachteile sind nur die, dass sie zur unbedingten Voraussetzung eine ziemlich beträchtliche Reklination des Kopfes hat und daher an Geduld und Ausdauer des Patienten Ansprüche stellt, denen leider vielfach nicht entsprochen werden dürfte.

Was leisten uns nun beide Methoden? Um dies $z u$ untersuchen, ist es unbedingt nötig, die verwandten Begriffe „Stützen", „Entlasten" und „Extendieren" scharf zu begrenzen. Das Verwandte von allen dreien ist, dass es sich stets um die Bekämpfung eines Druckes (oder Zuges) handelt; der Unterschied ist nur ein gradueller. Beim Stützen wird die positive Druckoder Zuggrösse vermindert, beim Entlasten wird sie auf 0 reduziert und beim Extendieren wird sie in eine negative Grösse umgewandelt. Die HinterhauptKinnstütze kann nun weder extendiren noch entlasten; und doch spricht ihre allgemeine Verbreitung dafür, dass ihre Vorteile nicht geringe sind. Einmal fixiert sie die Halswirbelsäule in genügendem Grade, indem sie die Rotation und Beugung des Kopfes nur ganz minimal zulässt. Zweitens gestattet sie, dem Halse eine beliebige Reklination zu geben. Drittens aber

1) Zeitschrift f. ortbopäd. Chirurgie, X. Bd. 1902. 
wird dem Kopfe ein bequemes Ruhelager geboten, zumal wenn die Stütze nicht starr angebracht ist, sondern federt (etwa durch Gummiring); dadurch wird der Cervikalabschnitt gewissermassen temporär entlastet und seinem allzu erheblichen Zusammensinken vorgebeugt. Die Hinterhaupt-Stirnstütze dagegen entlastet nicht nur, sondern sie kann auch direkt extendieren; von einem bequemen Ruhelager kann dabei freilich nicht die Rede sein.

Aber nicht nur zum Träger des Kopfes, auch zum Träger des Brustkorbes und dessen Eingeweide ist die Wirbelsäule. bestimmt. Hierzu kommt noch, dass am Thorax Schultergürtel und Arme befestigt sind, so dass das Gewicht, mit dem die Wirbelsäule in aufrechter Stellung belastet wird, wahrlich kein geringes ist. Nun könnte es auf den ersten Blick scheinen, als ob der Thorax selbst einen willkommenen Stützpunkt für die Wirbelsäule darböte. Sind aber die Rippen wirklich im stande, einen aus irgend welchem Grunde auf sie ausgeübten Druck auf die Dauer auszuhalten? Aus der Pathologie der Skoliose wissen wir, mit welch' erschreckender Leichtigkeit die Rippen dem Drucke der gegen sie andrängenden Wirbelsäule nachgeben und sich in der für diese Verkrümmung charakteristischen Weise auf der Seite der Konvexität ausbiegen zum Rippenbuckel, während auf der anderen Seite ihr normaler Bogen abgeflacht wird. Das hat seinen natürlichen Grund in dem lockeren Gefüge des Brustkorbes und in dem gracilen Bau jeder einzelnen Rippe. Denn der Thorax ist kein starrer fester Panzer, sondern jeder Atemzug verändert seine Gestalt und benötigt dazu eines Gehäuses, das leicht an Gewicht und beweglich in seinen Fugen ist. Ein dauernder Druck also, der auf eine Seite des Thorax ausgeübt wird, muss notgedrungen eine Verkrümmung der normalen Rippenbogen zur Folge haben.

Aber sehen wir von dem seitlichen Druck einmal ab; wäre es nicht möglich, den Thorax durch eine geeignete Stütze von den unteren seitlichen Partien aus in toto in die Höhe zu heben und so die Wirbelsäule zu entlasten? Auf den ersten Blick erscheint dies sehr verlockend. Der Rumpf eines nackten Menschen zeigt deutlich, dass von der Taille aus eine Verbreiterung nach oben - zum Schulterrand geht: darnach müsste es ja möglich sein, durch einen Apparat, der sich auf die Hüften stützt, den ganzen darüber gelegenen Rumpfabschnitt in die Höhe zu drücken, wobei freilich die Atmung nur abdominell erfolgen könnte. Leider aber ist dies aus dem einfachen Grunde unmöglich, weil das knöcherne Gerüst des Brustkorbes gar nicht einen sich nach oben verbreiternden Keil darstellt; der Thorax verbreitert sich vielmehr gerade umgekehrt von oben nach unten und das Gegenteil wird nur vorgetäuscht durch die an beiden Seiten des Thorax sich anhaftenden Schulterblätter und die zu ihnen sowie zum Oberarm ziehenden mächtigen Muskelmassen, die rorn und hinten den Übergang des Rumpfes in den Arm darstellen und zwischen sich die Achselhöhle fassen.

Wie man nun auf den Gedanken gekommen ist, die Achselhöhle als Stützpunkt für den Thorax heranzuziehen, ist absolut unverständlich; dass diese nie und nimmer dazu verwandt werden kann, liegt auf der Hand. Denn einmal sollen Muskeln - und namentlich so wichtige wie Pectoralis 
und Latissimus - vor jedem Drucke behütet werden; dann aber ist das Schulterblatt mit den Rippen nur lose verbunden und beantwortet jedes stärkere Andrängen einer Achselkrücke mit einfachem Hochziehen der Schulter, um so dem lästigen Drucke auszuweichen. Wenn man glaubt, durch Achselkrücken die Wirbelsäule strecken und den Thorax in die Höhe richten zu können, so verrät dies eine absolute Unkenntnis mit den gröbsten anatomischen Beziehungen zwischen Schulter und Brustkorb. Und doch: an wie vielen Korsetts wird man diesem Unfug begegnen! Lorenz sagt treffend, man wisse nicht, worüber man sich mehr wundern solle, ob über die Geduld der Patienten oder die Indolenz des Plexus brachialis. Wie viele meiner Patienten atmeten erleichtert auf, als ich sie von dieser ganz unnützen Qual befreite. Darum stellen wir die Entfernung der extendierenden Achselkrücke an Korsetts als Postulat des gesunden Menschenverstandes auf.

Wir müssen uns mit der Erkenntnis begnügen, dass weder Thorax noch Achselhöhle einen geeigneten Stützpunkt

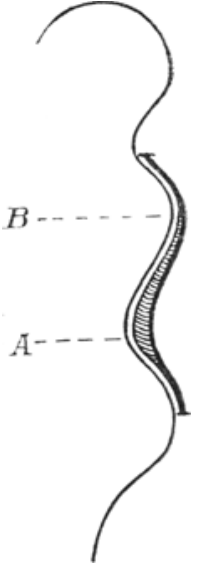

Fig. 1. für die Wirbelsäule darstellen. Diese ist vielmehr nur von einer Seite, von hinten, einer direkten therapeutischen Einwirkung zugängig. Allerdings ist damit unter normalen Verhältnissen nicht viel gewonnen, denn die mit Weichteilen ausgefüllte Dornfortsatzlinie stellt eine ziemlich glatte Fläche dar, bei der wir höchstens den etwas nach hinten vortretenden oberen Abschnitt der Lumballordose stützen können. Hiervon machen wir im täglichen Leben beim Sitzen Gebrauch. Wir empfinden diejenige Sitzgelegenheit als bequem, die durch ein geeignetes Polster die physiologische Lendenkrümmung ausfüllt und uns so vor einem Ermüdungsgefühl schützt, wie es sich zumal bei längerer Eisenbahnfahrt bemerkbar macht, wo durch die andauernde Erschütterung.die Rückenmuskulatur stark in Anspruch genommen wird. Wirkt in diesen Fällen die Unterstützung des "hohlen Kreuzes" schon angenehm, um wie viel mehr in allen Fällen von pathologisch vermehrter Lordose, bezw. Kyphose. Hierher gehört in erster Linie der Pottsche Buckel, die bei weitem häufigste Form einer Verkrümmung in sagittaler Ebene. Wir haben hier so ziemlich den einzigsten Fall, wo wir die Wirbelsäule direkt stützen können und man sollte

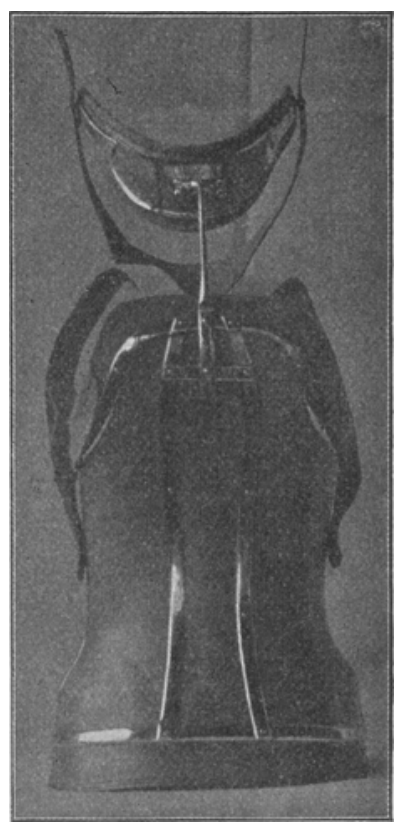

Fig. 2. meinen, dass sich kein KorsettKonstrukteur diese Gelegenheit entgehen lassen würde. Aber grade das Gegenteil ist der Fall: weder die Hessingschen Drellkorsetts, noch die im Rücken geteilten Korsetts sind im stande, diesen 
Zweck einwandsfrei zu erfüllen. Stellen wir uns das Profil eines Kyphotischen vor: B (Fig. 1) sei der Scheitel des Buckels, A der der kompensierenden Lordose (Fig. 2). Daneben ist das Profil des zu verordnenden Korsetts angedeutet. Unbedingte Voraussetzung desselben muss sein, dass es in den Hüften gut eingearbeitet ist und sich fest und unverschieblich auf die Darmbeinkämme aufstützt. Denn dieser Teil des Korsetts hat den Gegendruck gegen das vom Buckel nach abwärts gedrängte Polster auszuhalten. Den Teil des Korsetts nun, der dem Wirbelsäulenabschnitt $\mathrm{AB}$ entspricht, haben wir mit einem guten Polster zu versehen, denn gegen diesen Teil muss die Wirbelsäule bei ihrem Bestreben, der Eigenschwere folgend weiter zusammenzusinken, in erster Linie drücken. Auf die näheren Einzelheiten kommen wir noch später zurück.

Wenn es bei den Spondylitis-Korsetts lediglich auf die stützende Wirkung ankäme, so brauchten sie nicht weiter nach oben zu reichen als bis zur Buckelhöhe B; denn der ganze oberhalb von $\mathrm{B}$ gelegene Abschnitt

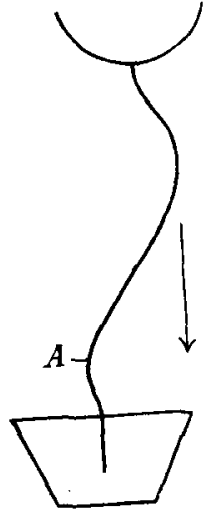

Fig. 3. stützt nicht im geringsten. Wohl aber erfüllt dieser eine zweite Forderung, nämlich die Fixation der Wirbelsäule, namentlich gegen seitliche Schwankungen. Je länger nun die Strecke AB ist, d. h. je höher die Kyphose sitzt, um so besser wird das Korsett stützen. Lumbalkyphosen liefern die allerungünstigsten mechanischen Verhältnisse, allerdings nicht nur wegen der Kürze der Strecke $A B$, sondern namentlich deshalb, weil hier die physiologische Lordose nicht nur nicht verstärkt, sondern sogar ins Gegenteil umgekehrt wird und dies ist einer der Gründe, weshalb hier auch die bestgearbeiteten Korsetts häufig völlig versagen.

Dies ist in grossen Zügen der anatomische Unterbau, auf dem wir die zweite Frage aufbauen: wann verschreiben wir orthopädische Korsetts und wie können wir den an sie gestellten Anforderungen gerecht werden? Man kann vier Indikationen für die Korsettherapie unterscheiden, je nachdem bezweckt wird

1. die Fixation,

2. die Stützung bezw. Entlastung,

3. die Extension,

4. die Redression der Wirbelsäule.

Am einwandfreiesten können wir der ersten Indikation, der Fixation, genügen, wenn es sich darum handelt, die Wirbelsäule in ihrem unteren Abschnitt festzustellen und ihre drei Bewegungen, Rotation, Seitenbeugung und antero-posteriore Beugung unmöglich zu machen. Hierzu gehört ein Korsett, das sich gut auf die Darmbeinkämme stützt, in der Taille genau anliegt, der Form der Wirbelsäule genau angepasst ist und nach oben bis ziemlich dicht unter die Achselhöhle geht, ohne jedoch die freie Bewegung des Pectoralis und Latissimus irgendwie einzuschränken. Ist die dorsale Thoraxdeformation so huchgradig, dass sie die Schulterblätter vom Thorax quasi abgehebelt hat, derart, dass die medialen Skapularränder nach hinten 
gerichtet sind, so ist es unter Umständen wünschenswert, auch die Schulterblätter möglichst zu fixieren. Hier ist mal ausnahmsweise die Achselkrücke indiziert, nur soll sie nicht das Schulterblatt hochheben, sondern dessen weiterer Dislokation nach vorne-aussen entgegenstreben. Zu diesem Zwecke wird das Collum scapulae durch das etwas nach innen gerichtete vordere Ende der Achselkrücke umfasst und nach rückwärts gedrängt.

Bedeutend schwieriger ist die Fixation des Cervikal- und oberen Dorsalabschnittes. Da der runde Hals uns keinen Stützpunkt zur Fixation darbietet, so muss das Korsett allemal mit einer Kopfstütze versehen werden, deren Mängel in mechanischer Beziehung wir bereits oben skizziert haben.

Alle fixierenden Korsetts müssen selbstverständlich Tag und Nacht getragen werden. Ein Punkt bedarf noch besonderer Erwähnung, das ist das An- und Ausziehen. Für die Fixation liegt darin entschieden ein gewisser Nachteil gegenüber den inamoviblen Rumpfverbänden. Doch kommt dies kaum in Betracht gegenüber dem grossen Vorteil einer erfrischenden Hautpflege und eines Wechsels der Trikotunterkleidung. Wird dieser Wechsel mit der nötigen Vorsicht vorgenommen, so ist die Gefahr eines Nachteiles für den Patienten nur ganz minimal. Darum ist den Angehörigen der SpondylitisKranken etwa folgende Anweisung mitzugeben: vor der Abnahme des Korsetts wird der Patient in einem Kopfhalter, der am besten von einer im Türrahmen angebrachten Rolle herabhängt, soweit vorsichtig und allmählich suspendiert, dass die Fusspitzen noch eben den Boden berühren. Die Oberschenkel werden von einer dritten Person festgehalten, um das Hin- und Herschwanken des suspendierten Körpers zu vermeiden, oder das Becken kann auch durch einen Gurt an einem Querbrett fixiert werden. Jetat erst wird das Korsett abgenommen, Brust und Rücken mit grüner Seife - ich selbst benutze dazu die vorzügliche Schleichsche Marmorseife - eingerieben. Man wäscht dieselbe zuerst mit warmem, dann mit kaltem Wasser ab und bestreicht nach vorsichtigem Abreiben gerötete Hautpartien, namentlich stets die Spinae, mit einer reizlosen Salbe. Hierauf wird der frische Trikot angezogen - falls dazu Trikotschlauch genommen wird, der sich wegen seines straffen, faltenlosen Anliegens sehr gut dazu eignet, muss derselbe schon vor der Suspension über Kopf und Arme des Patienten gestreift werden -; dann wird das Korsett angelegt und auf dieselbe Entfernung wie vorher zugeschnürt. Mit des Suspension wird jetzt allmählich nachgelassen und der Patient anfs Sopha gelegt, wo er nach jeder Waschung ca. eine Stunde ruhen soll. Die ganze Prozedur darf keine Schmerzen verursacht haben!

Die zweite Indikation eines Korsetts, die St ützung oder deren höchster Grad, die Entlastung, geht mit einer Fixation bis zu einem gewissen Grade stets Hand in Hand. Nur ist die Fixation der umfassendere Begriff, insofern als jede Fixation auch zugleich stützt, aber nicht jede Stütze fixiert. Das beste Beispiel liefert eine frische und eine ältere Spondylitis. Im ersteren Falle beherrscht in der Regel die schmerzhafte Entzündung das Krankheitsbild; besteht die Krankheit jedoch mehrere Jahre, so haben wir die mit grosser Muskelschwäche verbundene Deformität vor uns, die einer Fixation 
kaum mehr bedarf, wohl aber einer Stützung. Bezüglich der therapeutischen Wirkung des Korsetts besteht hier das umgekehrte Verhältnis wie bei der Fixation: war diese leichter auszuführen bei tiefsitzenden Krankheitsherden, so sind dieselben für eine Stützung die ungünstigsten Fälle, die man sich denken kann und verweisen wir auf das bereits oben angeführte.

Während Fixation und Entlastung auf einzelne Teile der Wirbelsäule beschränkt werden können, muss die Extension stets auf die ganze Wirbelsäule wirken. Ein derartiger Effekt kommt nur in Betracht bei einer aggressiven Skoliosenbehandlung, wie sie zwar schon vorher von anderen versucht, aber am exaktesten und vollkommensten von $\mathrm{W} \mathrm{ullste} \mathrm{in} \mathrm{durchgeführt} \mathrm{wurde.}$ Alle Körper, die wir extendieren wollen, müssen wir an ihren beiden Enden angreifen, in unserem Falle also an Becken und Kopf. Wie wir oben sahen, ist dies nur möglich bei der Hinterhaupt-Stirnstuitze, während die HinterhauptKinnstütze nicht einmal eine völlige Entlastung, geschweige denn eine Extension bewirkt.

Die letzte und umstrittenste Indikation ist die Redression. Zunächst ist es selbstverständlich, dass bei Korsetts nur von ganz allmählicher Redression die Rede sein kann; deshalb kombiniert man auch meist eine forciertere Redression in besonderen Apparaten mit fixierenden Gipsverbänden und wendet Redressionskorsetts nur an, um das errejchte Resultat zu erhalten, also zur Nachbehandlung. Daneben aber gibt es auch noch eine Anzahl von Korsetts, welche allein eine Redression bewirken sollen. Sie kommen fast ausschliesslich für die Skoliose in Betracht und hier müssen wir unterscheiden die Redression der Wirbelsäulen- von der der Thoraxdeformität. Erstere besteht in den Anfangsstadien der Skoliose allein und es fragt sich, ob deren Redression erfolgreich durchgeführt werden kann. Jede Extension redressiert bis zu einem gewissen Grade und in den ersten Stadien der Skoliose können wir in der Regel durch Extension die vorhandene Krümmung völlig zum Verschwinden bringen. Wir werden aber niemals von diesem Mittel Gebrauch machen, denn in der Medizin und namentlich in der Orthopädie muss dem Grundsatz mehr Geltung verschafft werden, dass Krankheit und therapeutischer Erfolg stets in einem bestimmten gegenseitigen Verhältnis stehen müssen. Dies ist hier nicht der Fall: ein extendierendes Korsett ist eine solche Qual und eine geringe seitliche Krümmung der Wirbelsäule ist an sich etwas so Gleichgültiges für den Patienten - vorausgesetzt, dass durch geeignete Massage und gymnastische Behandlung Vorkehrung gegen eine Verschlimmerung getroffen wird - dass es grausam wäre, in diesem Falle von jenem Mittel Gebrauch zu machen.

Aber können wir nicht, ohne den weiten Umweg der Extension zu wählen, auf direktere Weise der Seitenkrümmung zu Leibe gehen? Diese Frage ist von äusserster Wichtigkeit, weil gerade hier die gröbsten Fehler gemacht werden. Sie muss auf das bestimmteste verneint werden! Denn wie sollen wir eine seitliche Ausbiegung der Wirbelsäule bekämpfen, ohne einen unerträglichen Druck in der Lendengegend, oder einen direkt schädlichen auf die weichen, nachgiebigen Rippen auszuüben. Wir würden dadurch 
höchstens den konvexseitigen Rippenbogen zwischen Wirbelsäule und Korsett zusammenpressen und dadurch das Zustandekommen eines Rippenbuckels direkt begünstigen, von anderen Nachteilen, auf die wir noch später kommen, ganz abgesehen.

Bei den späteren Stadien der Skoliose aber kommt zu der Wirbelsäulenverkrümmung noch die entstellende Thoraxdeformität hinzu, bestehend aus einem oft erschreckend hochgradigen hinteren (konvexseitigen) und einem geringeren vorderen Rippenbuckel. Hier vermag ich nun den absoluten Korsettgegnern nicht unbedingt zu folgen, denn es lässt sich nicht einsehen, warum nicht durch einen zweckmässig redressierenden Druck eine Abflachung der Deformität erfolgen könnte. Nur ist dabei folgendes zu beachten: jede Skoliose ist entstanden durch eine fehlerhaft wirkende Belastung. Will man also derselben zu Leibe gehen, so hat man zunächst die Causa efficiens zu beseitigen, $d . h$. man muss die Wirbelsäule entlasten; noch besser, wir steigern die Entlastung zur wenigstens teilweise redressierenden Extension. Dann erst ist es mit Aussicht auf Erfolg möglich, durch diagonalen Druck den vergrösserten schrägen Thoraxdurchmesser zu verkleinern und so die Deformität allmählich zu verbessern. Derartige Versuche sind gemacht worden und werden wir darüber weiter unten berichten. Auf andere Weise als durch Extension und diagonalen und zugleich detorquierenden Druck redressierende Wirkung erziehen zu wollen, ist absolut unmöglich. Und doch wird das heute noch auf alle mögliche Art versucht; namentlich schweben mir dabei die Korsetts vor, wo von einem hinteren Mittelstück schmale seitliche Pelotten ausgehen. Sie werden uns noch in fast allen Bandagistenschaufenstern angeboten und sind orthopädisch nicht mehr ernst zu nehmen, sondern lassen sich nur einreihen in die Rubrik des groben Unfugs.

Wenden wir diese allgemeine Indikation nun auf die speziellen klinischen Fälle an. Wir wollen die Deformitäten des Thorax, die eine Korsettherapie indizieren können, in 4 Gruppen einteilen, nämlich 1. die neurogenen, 2. die rhachitischen, 3. die spondylitischen und 4. die habituellen. Zur neurogenen Gruppe gehören vor allem die paralytischen Deformitäten, meist Folge einer voraufgegangenen Poliomyelitis; ferner möchte ich hierher die Fälle von Tabes einreihen, die wegen Rückenschwäche und -Schmerzen einer Korsettbehandlung bedürfen. Endlich gehören hierher die dystrophischen Zustände, die zu einer Wirbelsäulenverkrümmung führen, also vor allem die Dystrophia musculorum progressiva, die in der Regel mit starker Lordose verbunden ist. Die Indikation, die Wirbelsäule zu stützen, ist hier eine so absolute, dass etwaige schädliche Folgen eines Stützapparates gar nicht in Betracht kommen. Das Stützen ist freilich meist nicht leicht, da selten eine Deformität vorhanden ist, die uns einen guten Angriffspunkt darböte; man muss sich daher oft damit begnügen, den Thorax zu fixieren und ihn so vor weiterem Zusammensinken, namentlich nach der Seite, schützen. Nur eine etwa vorhandene pathologische Lordose lässt eine bessere Stützung durch ein gutes Rückenpolster zu. Zu erwähnen ist noch ein Korsett für Tabiker, das wir selber zwar noch nicht auszuproben Gelegenheit hatten, das aber recht 
praktisch zu sein scheint: es ist von $\mathrm{Bade}^{2}$ ) angegeben und besteht aus zwei ledernen Teilen, die in der Lendengegend durch ein Scharnier verbunden sind und so eine seitliche Bewegung gestatten. Auch zeichnet es sich dadurch vorteilhaft vor anderen aus, dass es jeder Achselkrücke entbehrt.

Die rhachitischen Deformitäten unterscheiden sich für die Therapie von den habituellen dadurch, dass bei ihnen das Primum nocens nicht in der geschwächten Rückenmuskulatur, sondern im Knochen selbst zu suchen ist; ferner dass dasselbe bei kräftiger Ernährung etc. mit der Zeit schwindet, während die Muskelschwäche, wenn sie einmal zur pathologischen Haltung geführt hat, ohne zweckentsprechende Behandlung immer hochgradiger zu werden pflegt. Hat man es also mit einer drohenden oder bereits beginnenden rhachitischen Deformität zu tun, so wird man, falls aus Rücksicht auf die unteren Extremitäten keine Bettruhe erforderlich wird, die Kinder in Rumpfverbände auf $1 / 4-1 / 2$ Jahre stecken und während dieser Zeit seine Hauptsorge auf kräftige Diät und frische Luft richten. Nach dieser Zeit wäre die durch die lange Fixation geschwächte Muskulatur durch energische Massage und Gymnastik zu kräftigen. Leider kommen aber in der Regel die Kinder erst bei stärkerer Deformität zur Behandlung und da ist dann bis zu der Zeit, wo der rhachitische Prozess erfahrungsgemäss meist erloschen ist, also bis zum 6. Lebensjahre, das Tragen eines Stützkorsetts neben der anderen Behandlung wünschenswert, um noch $\mathrm{zu}$ retten, was zu retten ist. Ist die Rhachitis ausgeheilt, so ist die zurückgebliebene Deformität wie eine habituelle zu behandeln.

Allen Entzündungen der Wirbelsäule ist gemeinsam das Streben nach Fixation, welche in Kyphose erfolgt. In den nicht tuberkulösen Fällen, also z. B. bei Spondylitis traumatica, rheumatica und deformans werden wir durch ein frühzeitig verordnetes Korsett, das stützt und fixiert, den Grad der Kyphose sehr einschränken können. In vielen Fällen wird man dabei eine Kopfstuitze nicht entbehren können. Namentlich sei hier auf die rheumatischen Altersspondylitiden hingewiesen, welche sich selbst überlassen zu so hochgradigen Cervikalkyphosen führen können, dass das Kinn einen Decubitus auf dem Brustbein erzeugt. Hier heisst es: principiis obsta! Sobald die Halswirbelsäule ihre Reklinationsfähigkeit eingebüsst hat, verliere man die kostbare Zeit nicht mit Umschlägen oder Bäderkuren, sondern man schreite ungesäumt zur Anfertigung eines Korsetts mit Hinterhauptkinnstütze, denn das ist das einzige Mittel, um diesen Patienten ein erträgliches Alter zu verschaffen. Wird der Kopf nicht gestützt, so kann er oft schon nach kurzer Zeit auch mit äusserster Mühe kaum noch soweit gehoben werden, dass der Blick geradeaus ermöglicht wird.

Die weitaus häufigste Indikation liefert die tuberkulöse Spondylitis. In frischen Fällen dürfte das L o renzsche Reklinations-Gipsbett wohl das souveräne Mittel sein, von dem wir wenigstens so lange Gebrauch machen - müssen, als stärkere Schmerzen und Paresen bestehen. Senkungsabscesse

1) Bade, Zur Korsettbehandlung der Tabes dorsalis, Münch. Med. Wochenschrift 1901, Nr. 3. 
indizieren dagegen nicht immer eine absolute Ruhiglagerung, schon allein aus dem Grunde, weil sie oft erst längere Zeit nach Ablauf des akut-entzündlichen Stadiums auftreten und dann sehr langwierig zu sein pflegen. Glaubt man den Patienten aufstehen lassen zu können, so wird man als erste Stütze jedem Korsett den exakter fixierenden Rumpfgipsverband ev. mit Kopfhalter vorziehen und erst einige Monate später, wenn dem Patienten die ambulante Behandlung gut bekommen ist, zur Verordnung eines Korsetts schreiten. Dies soll in allererster Linie stützen und dadurch einen doppelten $Z$ weck erfüllen: das Gehen ermöglichen und den sich ausbildenden Gibbus nach Möglichkeit beschränken. Zweckmässig ist hier das von Jolling er angegebene Korsett, welches dadurch, dass es aus zwei seitlich zu schnürenden Teilen besteht, die Fixation des Rumpfes in einer dosierbaren Reklination ermöglicht. Doch ist seine Herstellung umständlicher, und dürften einfachere Stützkorsetts mit guter Kreuzpolsterung dieselben Dienste tun. Hat man den Kopf längere Zeit gestützt, so wird die Nackenmuskulatur schwach und atrophisch; um sie allmählich wieder zur Arbeit heranzuziehen, kann man den Kopf zwar noch stützen, aber ihm seine Bewegungen gestatten. Hierzu verwenden wir einen Kopthalter, welcher - ähnlich dem von Ipsen-Schede - nur eine hintere Stütze hat, die an ihrem oberen Ende mit einem Kugelgelenk versehen ist.

Vielumstritten ist die Indikation bei der letzten Gruppe, den habituellen Deformitäten. Während bei den drei vorhergehenden Gruppen im allgemeinen wohl Übereinstimmung unter den Orthopäden herrschen dürfte, tobt hier der Streit der Meinungen zwischen den absoluten Korsettgegnern und den Korsettoptimisten, die mit geradezu beneidenswerter Kritiklosigkeit jede Skoliose hoffnungsvoll einpanzern. Ich setzte dabei natürlich voraus, dass diese Korsetts wenigstens anatomisch und technisch richtig konstruiert sind und rede gar nicht von der grossen Zahl derer, die zwar einen hübschen Gewinn für den Verfertiger, aber absolut keinen für den Patienten darstellen. Man bedenke stets, dass bei allen habituellen Deformitäten, welcher Theorie über ihre Entstehung man auch sonst huldigen wolle, ein Moment eine sehr wichtige Rolle, für mich sogar die Hauptrolle spielt: die Muskelschwäche. Dieselbe muss aber von jedem Korsett gefördert werden, da durch dieses ja das freie Spiel der Muskelkräfte völlig aufgehoben wird. Eine Scoliotica leichten und mittleren Grades, welche durch tägliche Massage und Gymnastik soweit gebracht ist, dass sie ihre pathologische Haltung selbst redressieren kann, wird sich natürlich nicht von jetzt ab andauernd gerade halten; aber sie wird sich von Tag zu Tag immer häufiger daran erinnern, wie sie sich eigentlich halten sollte und wird dabei jedesmal unwillkürlich eine Selbstredression vornehmen, bis ihr dieselbe in Fleisch und Blut übergegangen ist, und vor allem, bis sie bei derselben nicht mehr ermüdet. Dazu gehört freilich eine gute Dosis Geduld und Fleiss und als absolut notwendiges Adiuvans - Eitelkeit. Ein Korsett macht natürlich diese selbstredressierenden Bewegungen unmöglich, und dies ist der grosse Schaden, den es bei allen habituellen Deformitäten anstiftet; wie gering sein Nutzen ist, hat der anatomische Teil unserer Betrachtungen ergeben. 
Zwei Ausnahmefälle allerdings gibt es, wo u. E. ein Skoliosenkorsett angewendet werden soll oder darf. Zunächst gehören hierher die schwersten Fälle mit hochgradigem Rippenbuckel, schon allein von dem praktischen Gesichtspunkte aus, dass man hier eben nur noch nützen, aber nichts mehr schaden kann. Den Rippenbuckel stützen wir genau wie den Pottschen Buckel durch ein im Korsett angebrachtes Polster und können hier, wo wir in dem hochgradig deformierten Thorax einen genügenden Angriffspunkt haben, die Wirbelsäule in günstigen Fällen vor völligem Zusammensinken schützen.

Die zweite Indikation ist, wie wir von vornherein zugeben, sehr diskutabel; denn abgesehen von den eingangs erwähnten Bedenken können wir hier auch direkt durch seitlichen Thoraxdruck schaden. Sie betrifft die Gruppe, bei der die grösste Krümmung in den Lendenabschnitt fällt und wo die auf der konkaven Seite stark vorstehende Hüfte und die oft bis zur Faltenbildung zusammengesunkene Taille das ganze Symptomenbild beherrscht. Diese Form spottet deshalb oft der intensivsten Behandlung, weil hier der ganze oberhalb des Scheitelpunktes A der Lumbalskoliose gelegene Rumpfabschnitt plus Arm quasi als einarmiger Hebel wirkt, und zwar im Sinne einer stetigen Zunahme der Verkrümmung, um so intensiver, je weniger die kompensierende Dorsalskoliose ausgebildet ist. Hier wird ein exakt anliegendes festes Korsett dem zur Seite neigenden Thorax als Strebepfeiler dienen; es muss aber zu dem Zwecke ein ziemlich bedeutender seitlicher Druck auf den Thorax ausgeübt werden, wodurch die Gefahr eines entstehenden Rippenbuckels bezw. der Vergrösserung eines bereits vorhandenen gegeben ist. Wir miissen also hiermit stets rechnen und in jedem einzelnen Falle entscheiden, ob hier der Vorteil oder der Nachteil eines Korsetts grösser sein wird.

Oft wird noch als Indikation eines Skoliosenkorsetts die bestehenden Rückenschmerzen angeführi. Diese sind lediglich Ermüdungsschmerzen der Muskulatur und gehen auf Massage und Gymnastik stets in kurzer Zeit zurück. Wir haben wenigstens noch keinen Fall gesehen, bei dem es nicht gelungen wäre, hierdurch die Schmerzhaftigkeit zu beseitigen. Sollte dies wider Erwarten einmal nicht eintreten, so läge der Verdacht einer beginnenden tuberkulösen Spondylitis nahe.

Zum Schluss noch ein kurzes Wort über die Konstruktion der Korsetts. Grundsätzlich zu verwerfen sind alle Korsetts, die ihr Heil in einer stützenden Armkrücke suchen: das sind in allererster Linie die Hessingschen Korsetts. Man lasse sich nicht durch ihr elegantes Äussere bestechen, das sie, zumal sie gewöhnlichen Korsetts ähnlich sehen, namentlich bei Damen beliebt macht. Es ist nicht recht erklärlich, wie auch in den ersten orthopädischen Lehrbüchern diese Korsetts so gelobt werden. Denn ausser den Armkrücken fehlt ihnen überhaupt jedes stützende Prinzip. Dass die hinteren Längsschienen, die an ihrem oberen Ende nicht einmal fixiert sind, genügend stützen könnten, wird niemand im Ernste behaupten wollen. Das Korsett kann im ganzen einen gewissen Halt gewähren, aber nur in einem Falle: wenn es nämlich ausserordentlich fest zugeschnürt wird. Aber ganz abgesehen von dem Schaden, den es dann 
anrichtet, ist es klar, dass die Mehrzahl der Patienten, sich selbst überlassen, diese Schnürung nicht so fest anzieht, als es bei der Anprobe vorgesehen war und dass mithin auch der geringe Halt, den es gewähren kann, rein illusorisch wird. Wer ohne Armkrücken absolut nicht auszukommen glaubt, mag sie in Gottes Namen am Korsett anbringen, nur soll er sich davor hüten, sie allein zu verwenden oder gar die Schultern damit in die Höhe drängen zu wollen. Auch die hinten geteilten Korsetts halten wir nicht für zweckmässig, wenigstens in keinem der Fälle, in denen hinten in der Mitte die stützende Polsterung angebracht werden muss. Zudem ist gar nicht einzusehen, weshalb wir zweiteilige Korsetts verwenden sollen, so lange wir einteilige bequem und sicher herstellen können. Obschon man zu letzteren eine Menge der verschiedensten Materialien verwandt hat, sind allgemeiner verbreitet doch nur zwei Stoffe: die Celluloidacetonlösung und das Leder. Aus verschiedenen Gründen, die hier zu weit führen würden, fertigen wir nach mannigfachen Versuchen mit anderem Material nur noch Korsetts aus Rindsleder an, demselben, das zu Schienenhülsenapparaten verwandt wird. Allein ist dasselbe allerdings nicht widerstandsfähig genug und wird - ähnlich den Lorenzschen Celluloidplattenkorsetts - durch aufgenietete Stahlschienen verstärkt (vergl. Fig. 2). Das Binglersche Hornhautleder ist für einteilige Korsetts - wenigstens bei einigermassen deformiertem Thorax - absolut unbrauchbar, da es wegen seiner ausserordentlichen Zähigkeit so gut wie gar nicht gewalkt werden kann. Wir stehen hier zwar in direktem Widerspruch zu Gocht ${ }^{1}$ ), der Hornhautleder sehr empfiehlt; wer aber die Fig. 101 und 104 seiner orthopädischen Technik miteinander vergleicht - ein den Körperformen angepasstes Korsett aus Cellulose und ein nicht angepasstes aus Hornhaut wird darin nur einen Beweis für unsere Behauptung erblicken.

Wir nehmen den Gipsabguss stets in Suspension, die der Schwere der Deformität anzupassen ist; unmittelbar unterhalb des am stärksten prominierenden Teiles wird die Polsterung auf das Gipsmodell aufgenagelt. Auch die Anlegung des Korsetts geschieht in Suspension; sinkt dann nach Aufhören derselben der Thorax zusammen, so hat er in der entsprechend gepolsterten Korsettwand eine gute Stütze. Achselkrücken verwenden wir so gut wie nie; dasselbe gilt von Pelotten, Gurten und elastischen Zügen.

Neuerdings sind zwei bemerkenswerte Versuche veröffentlicht worden, am Skoliosenkorsett geeignete redressierende Vorrichtungen anzubringen. Nachdem man vorher schon vielfach bemüht war, durch meist völlig ungeeigneten Pelottendruck der Deformität direkt zu Leibe zu gehen, hat $\mathrm{Roth}^{2}$ ) in Budapest ein „Detorsions- und Redressionskorsett" angegeben, das, soviel sich aus der einer klaren schematischen Zeichnung leider entbehrenden Darstellung ersehen lässt, den vergrösserten diagonalen Thoraxdurchmesser durch geeigneten Druck auf den hinteren und auf den vorderen Rippenbuckel verkleinern soll. Ich selbst hatte vor zwei Jahren einen im Prinzip völlig gleichen Gedanken: ich wollte von einem um den Thorax gelegten Stahlreifen

1) Gocht, Orthopädische Technik, Stuttgart, Enke 1901.

2) Zeitschrift f. orthopäd. Chirurgie, X. Band, 1902. 
aus durch eine vordere und eine hintere Pelotte den vergrösserten Thoraxdurchmesser quasi zusammenschrauben. Von der Ausführung dieses Planes hielt mich jedoch die Erwägung ab, dass diese Bemühung umsonst sei, wenn die Wirbelsäule nicht zugleich durch eine Kopfstütze vollständig entlastet würde, wie bereits oben ausgeführt. Das ist aber mit solchen Unannehmlichkeiten für den Patienten verbunden, dass ich mich dazu nicht recht entschliessen konnte. Ich glaube, dass an diesem Mangel auch der Roth sche Plan scheitern wird, den ich vergleichen möchte jemandem, der den Ausfluss eines überlaufenden Gefässes zuzuhalten bemüht ist, anstait den zuführenden Wasserhahn abzudrehen.

Der zweite Versuch stammt von Wullstein; er ist u. E. anatomisch einwandsfrei und klinisch der grösste Fortschritt in der Skoliosentherapie. Hier ist das Skoliosenkorsett das, was es stets bleiben wird, nämlich nur ein Glied in der grossen therapeutischen Kette. Sein Verfahren besteht kurz gesagt in wechselweiser Gymnastik und Fixation des erreichten Resultates für mehrere Wochen-Monate in einem Gipsverband. Zur Redression und Verbandanlegung dient ein eigens konstruierter Apparat, der technisch wohl der vollkommenste unter allen $\mathrm{zu}$ diesem Zwecke angegebenen sein dürfte. Später wird ein Korsett angefertigt, das zum prinzipiellen Unterschied vom Rothschen Apparat Redression mit dauernder Kopfextension verbindet und dennoch eine Beweglichkeit der Lendengegend und des Kopfes ermöglicht. Auch diese Korsetts haben Armkrücken, welche aber nicht der Extension das besorgt ja die Kopfstütze — sondern der Reklination der Schultern dienen.

Ein Bedenken erweckt die Wullsteinsche Methode ebenso wie die früheren von $\mathrm{Schanz}$ u. a. angegebenen, das freilich nicht dem Verfahren, sondern der Krankheit zur Last fällt: die ausserordentlich lange Dauer der Behandlung, die sich in der Regel über mehrere Jahre hinzieht. In der Privatpraxis kostet diese Krankheit schlechterdings ein Vermögen an Geld und an Zeit, was um so schwerwiegender ist, als die Mehrzahl unserer Patienten dem schulptlichtigen Alter angehört. Daher liesse sich diese Behandlung bei der minder bemittelten Klasse nur durchführen, wenn die grösseren Städte sich zur Einrichtung städtischer orthopädischer Institute entschliessen könnten, die sich namentlich der Skoliosenbehandlung zu widmen hätten und in einer angegliederten Werkstätte exakt sitzende Korsetts und Schienenhülsenapparate für geringen Preis liefern könnten. 